\title{
Las relaciones entre "Educación e Inclusión Social" en Argentina y Brasil: las apropiaciones específicas de algunos debates e ideas educativos de la trama discursiva internacional circulante
}

\author{
Hernán Amar* \\ Cesar Tello** \\ Guillermo Bianchi*** \\ Gabriel Asprella***
}

\section{Resumo}

Este artículo describe y analiza, en un primer movimiento, algunas problematizaciones sobre las relaciones entre "Educación e Inclusión Social" enunciadas por el Programa de Educación Para Todos (EPT), coordinado por la UNESCO. En el segundo movimiento, argumentamos que la producción de las discusiones e ideas educativas planteadas por el EPT son traducidas según las tradiciones históricas particulares de las regiones y/o sus Estados nacionales. Para dar cuenta de las mediaciones y pasajes efectuados entre un fragmento discursivo educativo de lo internacional circulante, y sus apropiaciones locales latinoamericanas en la historia reciente, en términos de continuidades y rupturas (neoliberalismo-posneoliberalismo). Por último, en un tercer movimiento, enunciamos algunas críticas sobre las problematizaciones entre la educación inclusiva y la calidad de la educación, dado que los debates y políticas públicas actuales tanto a nivel global como local se reducen a temáticas derivadas del significante calidad educativa. Por el contrario, entendemos que estas discusiones deben atender a la ruptura de las significaciones actuales sobre el lugar y carácter del Otro en tanto diversidad (aún teñidas de una visión etnocéntrica) y avanzar en el reconocimiento de los sujetos en términos de justicia social, recuperando algunas nociones como las de igualdad, emancipación y subjetividad politica.

Palabras clave: Políticas; Educación; Inclusión.

* Professor da Universidad Nacional de Tres de Febrero, Buenos Aires, Argentina.

** Professor doutor da Universidad Nacional de Tres de Febrero, Buenos Aires, Argentina.

*** Professor da Universidad Nacional de Tres de Febrero, Buenos Aires, Argentina.

**** Professor da Universidad Nacional de Tres de Febrero, Buenos Aires, Argentina. 


\section{Relations between "Education and Social Inclusion" in Argentina and Brazil: the specific appropriations of some debates and educational ideas of the current international discourse plot}

\section{Abstract}

This article describes and analyzes in a first movement, some problematizations on relations between "Education and Social Inclusion" enunciated by the Education for All, coordinated by UNESCO. In the second movement, we argue that the production of educational ideas and arguments raised by the EFA are translated according to the particular historical traditions of the regions and/or their nationals. To account for mediations and passages made between an educational discourse fragment the international circulation, and Latin American local appropriations in recent history, in terms of continuities and ruptures (neoliberalism-postneoliberalism). Finally, in a third movement, we enunciate some reviews about the problematics between inclusive education and quality of education, given that the current public policy debates and both global and local levels are reduced to themes arising from the significant educational quality. On the contrary, we believe that these discussions should address the current breakdown of meanings about the place and nature of the Other as diversity (even dyed an ethnocentric vision) and advance the recognition of the subjects in terms of social justice, recovering some notions like equality, emancipation and political subjectivity.

Keywords: Politics; Education; Inclusion.

\section{Introducción}

Este artículo describe y analiza, en un primer movimiento, algunas problematizaciones sobre las relaciones entre "Educación e Inclusión Social" enunciadas por el Programa de Educación Para Todos (EPT), coordinado por la UNESCO.

En el segundo movimiento, argumentamos que la producción de las discusiones e ideas educativas planteadas por el EPT son traducidas según las tradiciones históricas particulares de las regiones y/o sus Estados nacionales. Para dar cuenta de las mediaciones y pasajes efectuados entre un fragmento discursivo ${ }^{2}$ educativo de lo internacional circulante, y sus apropiaciones locales latinoamericanas en la historia reciente, en términos de continuidades y rupturas: neoliberalismo-posneoliberalismo

En el tercer movimiento, enunciamos algunas críticas sobre las problematizaciones entre la educación inclusiva y la calidad de la educación, dado que los debates y políticas públicas actuales tanto a nivel global como local se reducen a temáticas derivadas del significante calidad educativa. Por el contrario, entendemos que estas discusiones deben atender a la ruptura de las significaciones actuales sobre el lugar y carácter del Otro en tanto diversidad (teñidas ambas nociones de una visión etnocéntrica y banal). Así proponemos trascender las nociones de equidad y de igualdad 
de oportunidades, para avanzar en el reconocimiento de los sujetos en términos de justicia social, incluso recuperando las nociones de igualdad, emancipación y subjetividad politica, y dentro de este marco revisar la pertinencia y vigencia del significante "inclusión" social y educativa.

\section{Primer movimiento}

Según Schriewer (2011), en las últimas décadas se produjo un proceso de internacionalización de las políticas e ideas educativas, pero también de sus particulares apropiaciones locales en términos de traducciones ${ }^{3}$. Entendemos que es en la estructura de las relaciones socio-históricas dialécticas entre lo global/regional/estato-nacional que puede comprenderse la producción y reproducción de discursos, prácticas y políticas públicas sobre "Educación e Inclusión Social".

Las relaciones entre "Educación e Inclusión Social" comenzaron a problematizarse con regularidad a partir de la década del noventa, mediante el impulso otorgado por el EPT producido y acordado en los congresos internacionales organizados en Jomtien (Tailandia, 1990) y Dakar (SENEGAL, 2000). Según Rambla y Curran (2012), en estos encuentros se debatió el rol y las funciones a desplegar por los países desarrollados, los organismos internacionales y las agencias de la sociedad civil implicados en cuestiones relativas a la educación: bajo la coordinación de la UNESCO, y con la presencia de varios organismos (PNUD, UNICEF, FAO, OIT, OMS, entre otros), escuelas, organizaciones como la Campańa Mundial de la Educación y países miembros de la OCDE acordaron en Dakar, entre otras, las siguientes metas para 2015:

Objetivo 1: Extender y mejorar la protección y educación integrales de la primera infancia, especialmente para los niños más vulnerables y desfavorecidos.

Objetivo 2: Velar por que antes del año 2015 todos los niños, y sobre todo las niñas $y$ los niños que se encuentran en situaciones dificiles, tengan acceso a una enseñanza primaria y obligatoria de buena calidad y la terminen. [...]

Objetivo 6: Mejorar todos los aspectos cualitativos de la educación, garantizando los parámetros más elevados, para conseguir resultados de aprendizaje reconocidos y mensurables, especialmente en lectura, escritura, aritmética y competencias prácticas (UNESCO, 2012).

La "educación inclusiva" se comprendió como la capacidad para respetar e interpretar las diversas trayectorias de los sujetos, garantizando el acceso a las "oportunidades educativas" y al conocimiento para la construcción ciudadana y la vida social (ACEDO; OPERTTI, 2012). La perspectiva de "Educación e Inclusión Social" fue problematizada de diversos modos a partir de Jomtien y Dakar. Estas problematizaciones, condicionadas por las restricciones y posibilidades históricas (políticas, económicas, sociales y culturales) transformaron y/o complejizaron el concepto en los documentos coordinados por la UNESCO. 
A partir de la década del ochenta, los agentes y agencias dedicados a la "educación inclusiva" desplazaron el énfasis hacia aquellos estudiantes con "necesidades especiales": la Conferencia Mundial sobre Educación para Todos en Jomtien (1990), las Normas uniformes sobre la igualdad de oportunidades para las personas con discapacidad (1993) y fundamentalmente la Declaración de Salamanca y marco de acción para las necesidades educativas especiales (1994) se convirtieron en las referencias orientadoras para la incorporación de estos estudiantes en las escuelas comunes dentro de las líneas o ejes operativos del EPT. Por último, a partir de 2005 se plasmaron algunos documentos como las Directrices sobre politicas de inclusión en la educación (2005 y 2009), y se organizó el evento de la $48^{\circ}$ Reunión de la Conferencia Internacional de Educación: ambos llevaron adelante la problematización de las relaciones entre "Educación e Inclusión Social" hacia la transformación integral de los sistemas educativos nacionales (ACEDO; OPERTTI, 2012).

Como sostiene Serna (2012), los "enfoques dominantes de la cuestión social" en la década del noventa, modelados por la racionalidad política neoliberal abordaron la mayoría de las problemáticas en términos de "transitoriedad", cuestiones a ser corregidas por las políticas públicas "compensatorias". Bajo estas orientaciones generales también se recomendó el diseño de políticas públicas sobre las relaciones entre "Educación e Inclusión Social" según los lineamientos de los acuerdos programáticos formulados por la UNESCO, los países de la OCDE y las agencias de la sociedad civil englobados en el EPT: tanto las sugerencias sobre la integración de personas con "necesidades especiales educativas", como las propuestas dirigidas a los sectores vulnerables de la población contenían el sesgo del abordaje microscópico, acotado y específico, desvinculado de los condicionamientos ejercidos por la estructura social y de la inserción en un sistema general de políticas sociales estato-nacionales universales y mutuamente complementarias.

Serna (2012) sostiene que los acuerdos pos Consenso de Washington, en cambio, criticaron el "fundamentalismo de mercado" y su imposibilidad de construir sociedades con crecimiento y equidad social, lo cual generó respuestas críticas en algunas agencias y agendas internacionales, al punto de comenzar a discursivizar cuestiones relativas al desarrollo con inclusión social y la distribución del ingreso desde una perspectiva combinada de políticas "neo-universales" junto con acciones focalizadas diversificadas.

Estos debates sobre cuestiones y políticas públicas sociales a nivel global en la última década, que impactaron también en las reflexiones sobre las relaciones entre "Educación e Inclusión Social" fueron re-contextualizadas en algunos países de América Latina. No puede obviarse en este proceso de traducción la reflexión crítica de los campos intelectuales locales y la emergencia de gobiernos nacionales "neodesarrollistas" y "nacional-populares" en la región que diseñaron e implementaron nuevas políticas públicas posneoliberales orientadas, como sostiene García Delgado (2006; 2013), hacia la construcción de modelos de desarrollo productivo con inclusión social. En términos de Cao y Laguado (2014) se observan dos propuestas alternativas al Consenso de Washington: 1) el "nuevo desarrollismo"; 2) la "tradición nacional-popular". 
Las relaciones entre "Educación e Inclusión Social" en Argentina y Brasil: las apropiaciones específicas de algunos debates e ideas educativos de la trama discursiva internacional circulante

El enfoque del "nuevo desarrollismo" (corriente de economistas heterodoxos) propone la recuperación de la centralidad del Estado en materia de intervención económica y social y con una preocupación novedosa: la búsqueda y el mantenimiento de los superávits gemelos (fiscal y comercial) para asegurar la soberanía nacional sobre la orientación de las políticas públicas de desendeudamiento externo, la reindustrialización orientada hacia el mercado interno y la exportación, y la inversión pública creciente en educación, ciencia y tecnología aplicadas a la innovación de los procesos productivos (CAO; LAGUADO, 2014). Por su parte, Bresser Pereyra (2007) plantea que a diferencia de la ortodoxia convencional con centralidad en el mercado, el "nuevo desarrollismo" reconoce al mercado y su dinamismo a la hora de generar innovación y competitividad, pero le asigna al Estado-nación el rol indelegable de planificar y orientar el desarrollo con inclusión social.

La otra alternativa al neoliberalismo latinoamericano es la tradición "nacional-popular”, que incorpora los fundamentos teórico-prácticos del nuevo modelo de desarrollo económico y social y las reflexiones sobre las relaciones desiguales de poder (CAO; LAGUADO, 2014). Para el "nacionalismo popular", los países de la región se encuentran insertos en una posición subordinada respecto al sistema-mundo capitalista global, y su posicionamiento la condena a marcados desequilibrios económicos, sociales y culturales.

Para Cao y Laguado (2014), estas dos corrientes presentan divergencias o tensiones entre sí. Una de las críticas más fuertes que el "nuevo desarrollismo" enuncia respecto al "nacionalismo popular" es la renuencia a conservar el superávit fiscal y la escasa predisposición en la selección de recursos humanos cualificados para la coordinación de los niveles del Estado. Respecto a este último punto, el "nacionalismo popular" argumenta que el "nuevo desarrollismo" privilegia el perfil tecnocrático de los funcionarios y dirigentes estatales, construyendo políticas públicas atravesadas por el sesgo economicista que detentan estos funcionarios-especialistas "asépticos" o "despolitizados".

En Argentina, en los primeros años de gobierno de Néstor Kirchner se privilegió el enfoque del "nuevo desarrollismo" para construir una alternativa político-económica a la racionalidad política neoliberal. Pero, a partir del primer mandato de Cristina Fernández de Kirchner, la acentuación "neodesarrollista" sumó los principios fundantes del "nacionalismo-popular", generando ciertas contradicciones y debates internos en sus filas (y, en algunos casos, rupturas). En Brasil, en cambio, la acentuación "neodesarrollista" primó sobre otro tipo de enfoques alternativos al modelo de acumulación económica neoliberal, como lo demuestran las políticas tendientes al restablecimiento de los equilibrios macroeconómicos vía reducción de la inversión social impulsado por el gobierno de Dilma Rousseff en 2014.

\section{Segundo movimiento}

En materia de política educativa estato-nacional, a partir de la sanción de la Ley de Educación Nacional n. 26.206 de 2006, los gobiernos nacionales kirchneristas en Argentina recuperaron algunas orientaciones, conceptos y metas sobre educación 
inclusiva modelados en los debates y programas educativos globales como el de Educación para Todos en sus planteos a partir de mediados de la década de dos mil, pero re-contextualizados según las tradiciones históricas nacionales y la especificidad del posicionamiento político-pedagógico del espacio "nacional-popular". Las relaciones entre "Educación e Inclusión Social", entonces, intentaron atravesar a todo el sistema educativo nacional desde un eje político-pedagógico transformacional integral, a diferencia de las políticas públicas sobre "educación inclusiva" de la década del noventa (diseñadas a partir de la Ley Federal de Educación N²4.195 de 1993) centradas exclusivamente en las "necesidades especiales" y luego en los "márgenes" de la sociedad a través del despliegue de políticas focalizadas como el Plan Social Educativo. En este sentido, según Feldfeber y Saforcada (2012), el Plan Social Educativo de la década del noventa fue sustituido a partir del gobierno de Néstor Kirchner por el Plan Integral para la Igualdad Educativa, concibiendo a la educación igualitaria como una de las dimensiones de la igualdad social.

Estas políticas públicas posneoliberales sobre "educación inclusiva" fueron insertas, además, en un sistema general y articuladas con otras políticas sociales estatonacionales "neo-universales" como la Asignación Universal por Hijo (AUH), aunque sin dejar de responder con algunas intervenciones focalizadas diversificadas (Feldfeber y Saforcada, 2012). Todas estas disposiciones fueron dirigidas a apuntalar el acceso, la permanencia y/o el egreso de la escuela secundaria obligatoria (desde Inicial hasta la finalización del nivel medio), y no redujeron recursos del Estado Nacional aún a riesgo de incrementar la obsesión de los economistas "ortodoxos": el "gasto público" social.

Al respecto, Poggi (2014) destaca los avances en materia de políticas públicas educativas en sus relaciones con la inclusión social en Argentina, fundamentalmente en la ampliación/extensión de la escolarización del nivel Inicial y en la terminalidad de la escuela secundaria obligatoria (dos orientaciones planteadas en términos de superación de las metas y prioridades establecidas por el Programa de Educación para Todos, centradas fundamentalmente en la cobertura universal de la educación primaria). Aunque persistan, pese a este aumento global en todos los segmentos de edad, diferencias estructurales entre el nivel socioeconómico alto, medio y bajo (POGGI, 2014):

Tabla 1: Tasa de escolarización según nivel educativo en \%. Argentina, 2000-2010.

\begin{tabular}{l|c|c|c|c|c}
\hline \multicolumn{5}{c}{ Tasa de escolarización según nivel educativo en \%. Argentina, 2000-2010. } \\
\hline & 5 años & 6 a 11 años & 12 a 14 años & 15 a 17 años & 18 a 24 años \\
\hline 2000 & 73,7 & 99,2 & 97,8 & 85,2 & 45,4 \\
\hline 2010 & 93,6 & 99,5 & 97,4 & 88,3 & 47,2 \\
\hline Diferencia & $\mathbf{1 9 , 9}$ & $\mathbf{0 , 3}$ & $\mathbf{- 0 , 4}$ & $\mathbf{3 , 1}$ & $\mathbf{1 , 8}$ \\
\hline
\end{tabular}

Fuente: Poggi, 2014.

Por otro lado, si tomamos a los jóvenes de 20 ańos con secundario completo por nivel socioeconómico (NSE) también encontraremos un incremento considerable según el nivel socioeconómico en la comparación 2000-2010 (POGGI, 2014): 
Tabla 2: Jóvenes de 20 años con secundario completo por NSE, Argentina 2000-2010.

Jóvenes de 20 ańos con secundario completo por NSE, Argentina 2000-2010

\begin{tabular}{l|c|c|c}
\hline & NSE bajo & NSE medio & NSE alto \\
\hline 2000 & 35,3 & 54,1 & 76,3 \\
\hline 2010 & 48,4 & 68,9 & 75,5 \\
\hline Diferencia & $\mathbf{1 3 , 1}$ & $\mathbf{1 4 , 8}$ & $\mathbf{- 0 , 8}$ \\
\hline
\end{tabular}

Fuente: Poggi, 2014.

Los gobiernos nacionales de Lula da Silva y Dilma Rousseff, aunque desde una perspectiva centrada en la vertiente del "nuevo desarrollismo" recuperaron también algunos debates e ideas de los programas educativos internacionales como el de Educación para Todos bajo ciertos principios construidos en la época del pos Consenso de Washington, pero tradujeron las políticas según las tradiciones históricas de Brasil. Pese a las restricciones presupuestarias planteadas en términos político-económicos de los últimos años, que tienen su impacto y correlato en la expansión de las políticas públicas sociales, y a problemáticas político-pedagógicas pendientes diferentes a las de Argentina (aún persisten altas tasas de analfabetismo), Brasil también impulsó sus esfuerzos de políticas públicas sobre "educación inclusiva", según Poggi (2014), hacia el aumento del acceso, la permanencia y el egreso de los niveles Inicial, Primario y Secundario, aunque con una contundente ampliación de la tasa de escolarización fundamentalmente en el nivel Inicial y un incremento considerable en la terminalidad del nivel Secundario según el estrato socioeconómico. Insertó, además, sus políticas públicas sobre las relaciones entre "Educación e Inclusión Social" en un sistema general de políticas sociales estato-nacionales "neo-universales" mutuamente complementarias (por ejemplo, con el Programa Bolsa Familia) con el objetivo de extender/ ampliar/multiplicar los efectos de la inclusión consensuados a nivel global.

Al respecto, Poggi (2014) resalta los avances en las políticas públicas educativas y sus relaciones con la "Inclusión Social" respecto a la escolarización en todos los niveles educativos en Brasil:

Tabla 3: Tasa de Escolarización según nivel educativo en \%. Brasil, 2000-2010.

Tasa de Escolarización según nivel educativo en \%. Brasil, 2000-2010

\begin{tabular}{l|c|c|c|c|c}
\hline & 5 años & 6 a 11 años & 12 a 14 años & 15 a 17 años & 18 a 24 años \\
\hline 2000 & 65,9 & 95,5 & 95 & 81,1 & 34 \\
\hline 2010 & 86,6 & 98,4 & 97,9 & 83,7 & 28,9 \\
\hline Diferencia & $\mathbf{2 0 , 7}$ & $\mathbf{2 , 9}$ & $\mathbf{2 , 9}$ & $\mathbf{2 , 6}$ & $\mathbf{- 5 , 1}$ \\
\hline
\end{tabular}

Fuente: Poggi, 2014.

Respecto a los jóvenes de 20 años con secundario completo según el nivel socioeconómico, Poggi (2014) demuestra que se han evidenciado avances significativos en todos los estratos en Brasil: 
Tabla 4: Jóvenes de 20 años con secundario completo por NSE, Brasil 2000-2010.

\begin{tabular}{l|c|c|c|}
\cline { 2 - 4 } & \multicolumn{3}{|c|}{ Jóvenes de 20 años con secundario completo por NSE, Brasil 2000-2010 } \\
\hline 2000 & NSE bajo & NSE medio & NSE alto \\
\hline 2010 & 19,8 & 40,1 & 67,5 \\
\hline Diferencia & 41,2 & 58,4 & 80,7 \\
\hline
\end{tabular}

Fuente: Poggi, 2014.

\section{Tercer movimiento}

A pesar de estos avances, Poggi (2014) también enuncia los problemas que presentan las políticas de "educación inclusiva" en la región respecto a lo que se denomina "calidad de la educación": entre otras cuestiones, los "bajos" conocimientos detentados por nuestros estudiantes, y que se manifiestan en algunas evaluaciones como el Segundo Estudio Regional Comparativo y Explicativo (SERCE 2010), ${ }^{4}$ y en pruebas internacionales como PISA. ${ }^{5}$

Algunos intelectuales, especialistas y funcionarios del campo educativo cuando se refieren a "educación inclusiva" la circunscriben sólo a comparaciones en términos de "rankings" entre países, vinculándola a la calidad de la educación y como una cuestión meramente instrumental. En este sentido, para pensar y problematizar la noción de "inclusión" social y educativa evitando las sedimentaciones ideológicas neoliberales que conlleva, consideramos que estas discusiones deben atender a la ruptura de las significaciones actuales sobre el lugar y carácter del Otro y sobre diversidad (teñidas ambas nociones de una visión etnocéntrica y banal). Debemos trascender las nociones de equidad y de igualdad de oportunidades, para avanzar en el reconocimiento de los sujetos en términos de justicia social, incluso recuperando las nociones de igualdad, emancipación y subjetividad política.

Las ideas de lo que en este trabajo llamamos "tercer movimiento" plantean una construcción en proceso y en tensión. Argumentamos que no asumimos la justicia social como aquella sobre la que se ha teorizado en los últimos cien años: una teoría de la justicia social liberal, impulsada particularmente por las reflexiones de John Rawls. Para Rawls (1993), la tesis de la prioridad del principio de libertad supone la interpretación de la libertad como valor absoluto y, consecuentemente, la interpretación de la necesidad como valor relativo. Más aún, las posiciones liberales se apuran a colocar en el lugar principalísimo de la libertad el derecho de propiedad, aún a cuenta de excluir y limitar otras libertades, derechos y necesidades, y entienden la libertad casi exclusivamente en un sentido "negativo" como ausencia de obstáculo y coerción sobre la acción, y no como capacidad "positiva”, en el sentido de un poder, un deseo, una decisión: en fin, de la autodeteminación de una voluntad (BERLÍN, 1998). En nuestras reflexiones sobre estas cuestiones, y en particular en relación al concepto de justicia, nos hallamos más próximos o bien al marco conceptual de la justicia social en términos de la interpelación radical del Otro en tanto la problematizó Levinas (1980), o bien de la transformación subjetiva (individual y colectiva) a través del sosteni- 
miento de la afirmación de la igualdad como principio axiomático y del rechazo de la concepción de justicia asociada a la noción de víctima y de una subjetividad impotente (BADIOU, 2007). Reconociendo las tensiones teóricas y prácticas no resueltas entre políticas estato-nacionales y los postulados de la igualdad y la emancipación, tomadas como una cuestión no de "políticas sociales" sino de "subjetividad política", lo que Badiou (2009) denomina "política de la igualdad”, que consiste no sólo en un programa, sino también en una práctica sostenida por un sujeto político (colectivo).

\section{Reflexiones finales}

Argentina y Brasil, a pesar de las diferencias en sus acentuaciones "nacional -popular" y "neodesarrollista" respectivamente, presentan puntos de convergencia cuando deben traducir o re-contextualizar a nivel local ciertos debates e ideas sobre las relaciones entre "Educación e Inclusión Social"

Como hemos visto en este trabajo, las orientaciones de las propuestas educativas de alcance mundial del Programa de Educación para Todos, y sus pasajes mediados y traducidos en las políticas de "educación inclusiva" en ambos países de la región latinoamericana asumen nuevos sentidos.

Dado que algunos de los debates a nivel global/regional/nacional sobre la "educación inclusiva" abordan las cuestiones sobre la "calidad educativa" en términos meramente instrumentales, sostenemos que las relaciones entre "Educación e Inclusión Social" deberían problematizar otras cuestiones político-educativas, tales como la construcción de la subjetividad política, la recuperación del Otro y la radicalidad de sus interpelaciones, y la justicia social.

Por último enunciamos algunas críticas a la noción de inclusión educativa pero lo hacemos con un carácter propositivo considerando que la categoría de inclusión nos lleva a preguntarnos si la inclusión y la igualdad son una cuestión de políticas sociales o se trata de un gesto "generoso" de los sectores sociales beneficiados que a través del Estado autorizan o producen compensaciones para los sectores vulnerables y excluidos de la sociedad.

Ahora bien, para el lector desprevenido de los documentos que seńalamos en este artículo podrían considerarse "loables" en cuanto a la relación Estado y educación. Ahora bien, observamos una contradicción entre el carácter "progresista" de esas propuestas y recomendaciones y el horizonte de mejora que dicen promover pero que resultan negadas desde los dispositivos, relaciones y políticas económicas realmente promovidas desde los países centrales para los países periféricos. ¿Se trata de una contradicción entre esas políticas económicas que afectan la posibilidad de los discursos y políticas por una "inclusión" efectiva en términos educativos y sociales? ¿O se trata más bien de una contradicción en la noción misma de "inclusión”?

Se torna necesario un registro atento y crítico del significante "inclusión" desde nuestro lugar periférico ante la evidencia de distintas formas discursivas y programáticas. 
Otra cuestión sobre el significante de "inclusión educativa" consiste en señalar que "inclusión" educativa y social es un significante utilizado tanto en el marco de las políticas neoliberales de 1990 como dentro de los llamados estados posneoliberales. ¿¿Dice lo mismo "inclusión" cuando es usado en uno u otro contexto? ¿Varían las significaciones en términos de traducciones de esos significantes? Por otro lado debemos situar el foco en el lugar de enunciación de la inclusión. La figura del otro y la diversidad, retomado en las políticas compensatorias de los 80 hasta nuestros días, pierde la radicalidad de origen que tenía en la filosofía de Lévinas, y a diferencia del sentido y aspiración más profundo de la filosofía de aquel, revela un carácter etnocéntrico. El "otro", ahora es colocado en un lugar de falta y déficit. El sujeto (individual, colectivo, o a través de la representación estatal) que "incluye" ocupa el lugar central de la escena y es el que asigna y distribuye la igualdad. También aparece una noción empobrecida de diversidad, ya que el sujeto que incluye determina el grado de las diferencias aceptables (Quiero que seas como eres, pero del modo en que yo considero que se debe expresar tu diferencia). El otro radicalmente diferente es discriminado o estigmatizado. El sujeto enunciador del discurso de la inclusión determina el límite y el criterio de la inclusión y la exclusión, define los lugares ocupados y los merecimientos para la ayuda. "Quien establece la política compensatoria construye subjetivamente al merecedor de recibirla, pero sobre todo, en esa diferenciación entre unos y otros, se constituye subjetivamente a sí mismo, distinguiéndose del otro, porque, básicamente no lo considera un igual. Esta marca desigualitaria instalada en la médula de las relaciones entre humanos, no hay politica social ni educativa que pueda compensarla" (CERLETTI, 2010: 44-45).

Finalmente no podemos dejar de considerar que la imagen de la compensación remite a una figura jurídica que es la noción de víctima. Pero debe contemplarse que "una víctima no es un sujeto político", sino que remite a formas de impotencia que requieren de políticas compensatorias, que a su vez plantean "interrogantes prácticos". ¿La compensación, se debe tratar individual o colectivamente? ¿El desfavorecido, lo es por pertenecer a un grupo social, étnico, a un género?

Las acciones de discriminación positiva, cuando son entendidas de modo individual pueden entrar en conflicto con la discriminación "legítima" debida al mérito que promueven los mismos organismos o instituciones.

\section{Referencias}

ACEDO, C.; OPERTTI, R. Educación Inclusiva: de focalizar grupos y escuelas a lograr una educación de calidad como el corazón de una Educación para Todos. In: RAMBLA, X. (Edt.). La Educación para Todos en América Latina. Buenos Aires: Miño Dávila Editores, 2012.

BADIOU, A. Justicia, filosofía y literatura. Rosario: Homo Sapiens Ediciones, 2007.

Compendio de meta-política. Buenos Aires: Prometeo, 2009.

BERLIN, I. "Dos conceptos de libertad", en Cuatro ensayos sobre la libertad. Madrid: Alianza Universidad, 1988.

BRESSER PEREYRA, L. C. (2007) Estado y mercado en el Nuevo Desarrollismo. Revista Nueva Sociedad, n. 210, julio-agosto de 2007. Caracas, Venezuela, 2007.

CERLETTI, A. Políticas sociales y políticas igualitarias. In: GÓMEZ CAMARENA, C.; UZÍN OLLEROS, A. (Comp.). Badiou fuera de sus límites. Buenos Aires: Imago Mundi, 2010. 
Las relaciones entre "Educación e Inclusión Social" en Argentina y Brasil: las apropiaciones específicas de algunos debates e ideas educativos de la trama discursiva internacional circulante

CAO, H.; LAGUADO, A. Una administración pública para las sociedades posneoliberales en América Latina. Documento INAP (mimeo), Buenos Aires, 2014.

FELDFEBER, M.; SAFORCADA, F. Políticas educativas y derecho a la educación en Argentina:un análisis de las metas educativas en el nuevo escenariolatinoamericano. In: RAMBLA, X. (Edt.). La Educación para Todos en América Latina. Buenos Aires: Miño Dávila Editores, 2012.

DELGADO, D. G. Introducción. In: DELGADO, D. G.; NOSETTO, L. (Comps.). El desarrollo en un contexto postneoliberal. Hacia una sociedad para todos. Buenos Aires: CICCUS, 2006.

D. G.; FERRIER, M. C. R. El nuevo paradigma. Algunas reflexiones sobre el cambio epocal. Revista Estado y Políticas Públicas, Año 1, n. 1. octubre 2013. Buenos Aires, FLACSO Argentina, 2013.

LENDVAI, N.; STUBBS, P. Políticas como tradução: situando as políticas sociais transnacionais. Práxis Educativa, Ponta Grossa, v. 7, n. 1, p. 11-31, jan./jun, 2012.

LÉVINAS, E. El tiempo y el otro. Madrid: Paidós, 1993.

LEY FEDERAL DE EDUCACIÓN N²4.195/93.

LEY DE EDUCACIÓN NACIONAL N²6.206/06.

POGGI, M. La educación en América Latina: logros y desafíos pendientes. Buenos Aires: FundaciónSantillana, 2014.

RAMBLA, X.; CURRAN, M. La Educación para Todos en América Latina y en el mundo. In: RAMBLA, X. (Edt.). La Educación para Todos en América Latina. Buenos Aires: Miño Dávila Editores, 2012.

RAWLS, J. Teoría de la Justicia. Buenos Aires: FCE, 1993.

SCHRIEWER, J. Sistema mundial y redes de interrelación: la internacionalización de la Educación y el papel de la investigación comparada. In: CARUSO, M.; TENORTH, H. E. Internacionalización, políticas educativas y reflexión pedagógica en un medio global. Buenos Aires: Granica, 2011.

SERNA, M. La Educación para Todos: nuevos enfoques, viejos problemas de la cooperación internacional. In: RAMBLA, X. (Edt.). La Educación para Todos en América Latina. Buenos Aires: Miño Dávila Editores, 2012.

VERÓN, E. La semiosis social. Gedisa: Barcelona, 1993.

Fragmentos de un tejido. Gedisa: Barcelona, 2003.

\section{Anotaciones}

${ }^{1}$ Enunciamos las relaciones entre "Educación e Inclusión Social" para dar cuenta de algunas de las diversas problematizaciones sobre las contribuciones de la educación institucionalizada respecto a los procesos de inclusión social efectuadas a través de la producción de conocimiento en debates, ideas y políticas públicas educativas a nivel global, regional y/o estato-nacional. El uso de comillas tiene por intención recuperar, a través de la construcción de un significante-marco, las huellas discursivas de referencia sobre este eje temático materializadas en diversos discursos y prácticas político-educativos producidos por intelectuales, especialistas, expertos, funcionarios y planificadores.

2 En términos de Verón (1993 y 2004), el fragmento discursivo es una materia significante finita inserta en una red discursiva histórico-social, intertextual, sujeta a condiciones sociales productivas, tanto de producción como de reconocimiento.

${ }^{3}$ Es decir, en términos de Lendvai y Stubbs (2012) se pueden estar produciendo las mismas vinculaciones y no muchas transformaciones en términos de la configuración estatal en relación a la década de 1990. La diferencia podría radicar en cómo se producen las "traducciones" en términos de las políticas públicas en algunos países de la región latinoamericana. En este sentido, no se puede obviar que estas traducciones son transformadoras de la propia estructura de algunos de los Estados nacionales de la región.

${ }^{4}$ El SERCE fue una evaluación de Matemática y Lenguaje a los alumnos de tercer y sexto grado de la educación primaria.

${ }^{5}$ PISA es una evaluación realizada a los estudiantes de 15 ańos en Matemática, Lectura y Ciencias, con independencia del grado o ańo de estudio al que asisten. 
Hernán Amar - Cesar Tello - Guillermo Bianchi - Gabriel Asprella

\section{Correspondência}

Hernán Amar - Universidad Nacional de Tres de Febrero, Universidad Nacional de Tres de Febrero. Av. San Martín 2508 (B1678GQO). Caseros, 1900 - Tres de Febrero - Argentina.

E-mail: hamar@untref.edu.ar - cesargeronimotello@yahoo.com.ar - gbianchi@untref.edu.ar - gasprella@untref.edu.ar

Recebido em 29 de junho de 2015

Aprovado em 20 de agosto de 2015 